\title{
Laboratory measurements and astronomical search for cyanomethanimine ${ }^{\star}$
}

\author{
M. Melosso ${ }^{1}$, A. Melli ${ }^{1}$, C. Puzzarini ${ }^{1,2}$, C. Codella ${ }^{2}$, L. Spada ${ }^{1,3}$, L. Dore ${ }^{1}$, C. Degli Esposti ${ }^{1}$, B. Lefloch $^{4}$, \\ R. Bachiller ${ }^{5}$, C. Ceccarelli ${ }^{4}, 2$, J. Cernicharo ${ }^{6}$, and V. Barone ${ }^{3}$ \\ 1 Dipartimento di Chimica "Giacomo Ciamician", Università di Bologna, via F. Selmi 2, 40126 Bologna, Italy \\ e-mail: cristina.puzzarini@unibo.it \\ 2 INAF, Osservatorio Astronomico di Arcetri, Largo E. Fermi 5, 50125 Firenze, Italy \\ 3 Scuola Normale Superiore, Piazza dei Cavalieri 7, 56126 Pisa, Italy \\ ${ }^{4}$ Univ. Grenoble Alpes, CNRS, Institut de Planétologie et d'Astrophysique de Grenoble (IPAG), 38000 Grenoble, France \\ 5 IGN, Observatorio Astronómico Nacional, Calle Alfonso XII, 28004 Madrid, Spain \\ ${ }^{6}$ Grupo de Astrofísica Molecular. Instituto de CC. de Materiales de Madrid (ICMM-CSIC). Sor Juana Inés de la Cruz 3, Cantoblanco, \\ 28049 Madrid, Spain
}

Received 19 September 2017 / Accepted 16 October 2017

\begin{abstract}
Context. C-cyanomethanimine ( $\mathrm{HNCHCN}$ ), existing in the two $Z$ and $E$ isomeric forms, is a key prebiotic molecule, but, so far, only the $E$ isomer has been detected toward the massive star-forming region Sagittarius B2(N) using transitions in the radio wavelength domain

Aims. With the aim of detecting HNCHCN in Sun-like-star forming regions, the laboratory investigation of its rotational spectrum has been extended to the millimeter-/submillimeter-wave ( $\mathrm{mm}$-/submm-) spectral window in which several unbiased spectral surveys have been already carried out.

Methods. High-resolution laboratory measurements of the rotational spectrum of C-cyanomethanimine were carried out in the 100$420 \mathrm{GHz}$ range using a frequency-modulation absorption spectrometer. We then searched for the C-cyanomethanimine spectral features in the mm-wave range using the high-sensitivity and unbiased spectral surveys obtained with the IRAM 30-m antenna in the ASAI context, the earliest stages of star formation from starless to evolved Class I objects being sampled.

Results. For both the $Z$ and $E$ isomers, the spectroscopic work has led to an improved and extended knowledge of the spectroscopic parameters, thus providing accurate predictions of the rotational signatures up to $\sim 700 \mathrm{GHz}$. So far, no C-cyanomethanimine emission has been detected toward the ASAI targets, and upper limits of the column density of $\sim 10^{11}-10^{12} \mathrm{~cm}^{-2}$ could only be derived. Consequently, the C-cyanomethanimine abundances have to be less than a few $10^{-10}$ for starless and hot-corinos. A less stringent constraint, $\leq 10^{-9}$, is obtained for shocks sites.

Conclusions. The combination of the upper limits of the abundances of C-cyanomethanimine together with accurate laboratory frequencies up to $\sim 700 \mathrm{GHz}$ poses the basis for future higher sensitivity searches around Sun-like-star forming regions. For compact (typically less than $1^{\prime \prime}$ ) and chemically enriched sources such as hot-corinos, the use of interferometers as NOEMA and ALMA in their extended configurations are clearly needed.
\end{abstract}

Key words. ISM: molecules - molecular data - methods: data analysis - methods: laboratory: molecular

\section{Introduction}

Among the goals of astrochemistry, the detection of potential prebiotic molecules in astrophysical environments, and in particular in star forming regions, is fundamental in view of possibly understanding the origin of life. In recent years, several large programs have been devoted to the detection of prebiotic species: Prebiotic Interstellar MOlecular Survey (PRIMOS ${ }^{1}$ ) project with the NRAO Green Bank Telescope (GBT), the IRAS16293-2422 Millimeter And Submillimeter Spectral Survey² (TIMASSS)

\footnotetext{
$\star$ The complete list of the measured transitions for $Z$ - and $E$-C-cyanomethanimine is only available at the CDS via anonymous ftp to cdsarc.u-strasbg. fr (130.79.128.5) or via http://cdsarc.u-strasbg.fr/viz-bin/qcat?J/A+A/609/A121 1 http://www.cv.nrao.edu/PRIMOS/

2 http://www-laog.obs.ujf-grenoble.fr/heberges/timasss
}

with the IRAM 30-m and JCMT single-dishes, CHESS $^{3}$ (the Herschel Chemical Surveys of Star forming regions), $\mathrm{ASAI}^{4}$ (Astronomical Surveys At IRAM) with the IRAM 30-m antenna, and, more recently, SOLIS ${ }^{5}$ (Seeds Of Life In Space) with IRAM NOrthern Extended Millimeter Array (NOEMA), and PILS 6 (The ALMA Protostellar Interferometric Line Survey). These projects contributed to the census of a large number of new interstellar molecules (containing between six and eleven atoms), see for example the Cologne Database for Molecular Spectroscopy (CDMS; Müller et al. 2005).

Among the various chemical species, the compounds containing the $\mathrm{CN}$ moiety are considered prebiotic molecules as

\footnotetext{
3 http://www-laog.obs.ujf-grenoble.fr/heberges/chess

4 http://www.oan.es/asai/

5 http://solis.osug.fr/

6 http://youngstars.nbi.dk/PILS
} 
potential precursors of amino acids (see, for example, Balucani 2009, and references therein). The simplest one is HCN, which is ubiquitous in the interstellar medium (ISM). A particular case is represented by cyanomethanimine. Among the $\mathrm{HCN}$ dimers, the $Z$ - and $E$-C-cyanomethanimine forms (HNCHCN) as well as $\mathrm{N}$-cyanomethanimine $\left(\mathrm{CH}_{2} \mathrm{NCN}\right)$ are isomers more stable than two isolated HCN molecules (Evans et al. 1991). On general grounds, hydrogen cyanide dimers are thought to play a role as intermediates in the prebiotic synthesis of purines and proteins (Ferris \& Hagan 1984). Within this context, cyanomethanimine, and in particular its $\mathrm{C}$-form $(\mathrm{C}-\mathrm{HNCHCN})$ can be considered unique in the family of COMs, and its detection around Sun-like protostars would be crucial in understanding the prebiotic chemistry in regions that will form planetary systems.

C-cyanomethanimine has been detected by Zaleski et al. (2013) toward the massive star-forming region Sagittarius (Sgr) $\mathrm{B} 2(\mathrm{~N})$, placed at $8.5 \mathrm{kpc}$ from the Sun, within the PRIMOS context. To our knowledge, that reported by Zaleski et al. (2013) is the first and so far unique detection of interstellar HNCHCN in our Galaxy. The authors observed emission due to low-excitation ( $E_{\text {up }}$ up to $7 \mathrm{~K}$ ) transitions of the $E$ isomer in the $\simeq 9.5-48 \mathrm{GHz}$ spectral range.

The detection of HNCHCN toward Sgr B2(N) in the centimeter-wave spectral window calls for further searches at higher frequencies, in the $\mathrm{mm}$-/submm-wave spectral range, in regions forming future Sun-like stars using both singledishes, like the IRAM 30-m antenna, and interferometers, such as the IRAM NOEMA and Atacama Large Millimeter/submillimeter Array (ALMA). In addition, taking into account the increased sensitivities and new spectral windows offered by these telescopes (ALMA can touch the $\mathrm{THz}$ region), it might be interesting to extend the observation of rotational features at far higher frequencies than those of Zaleski et al. (2013). Laboratory studies for C-cyanomethanimine were indeed limited to the portion of rotational spectrum below $100 \mathrm{GHz}$ (Takeo et al. 1986; Takano et al. 1990; Zaleski et al. 2013). Because extrapolations from low-frequency laboratory measurements might provide inaccurate higher frequencies, the extension of the experimental investigation of rotational spectra of $Z$ - and $E$-C-cyanomethanimine is therefore warranted. To guide this extension to higher frequency, a preliminary computational investigation of the spectroscopic parameters was carried out (Puzzarini 2015), thus pointing out the effect of the centrifugal distortion terms as well as the limited reliability of the $A$ rotational constant for both isomeric species.

Furthermore, rotational spectra of both isomers show a maximum of intensity at frequencies higher than $100 \mathrm{GHz}$. As it will be shown later in the manuscript, the $E$ isomer shows strong $a$ - and $b$-type spectra $\left(\mu_{a}=3.25(5) \mathrm{D}, \mu_{b}=2.51(2)\right.$ D; Takano et al. 1990) even at low temperatures, whose maxima shift from $\sim 120$ and $\sim 450 \mathrm{GHz}$ at $T=10 \mathrm{~K}$ to $\sim 410 \mathrm{GHz}$ and $>1 \mathrm{THz}$ at $T=300 \mathrm{~K}$, respectively. $Z$-C-cyanomethanimine presents a weak $b$-type spectrum $\left(\mu_{b}=0.4(5) \mathrm{D}\right.$; Takano et al. 1990), while more intense (but still weaker than the $E$ one by about one order of magnitude) is the $a$-type one ( $\mu_{a}=1.35(10)$ D; Takano et al. 1990), whose maximum shifts from $\sim 100 \mathrm{GHz}$ at $T=10 \mathrm{~K}$ to $\sim 430 \mathrm{GHz}$ at $T=300 \mathrm{~K}$.

The sensitivity reached in absorption measurements against the strong continuum source SgrB2 by Zaleski et al. (2013) cannot be obtained toward dark clouds because of the strong continuum emission itself, which is more than a factor of ten larger than the kinetic temperature of quiescent clouds. However, mm line emissions are the best tracers for detecting $\mathrm{HNCHCN}$, or to provide significant upper limits to its abundance, in dark clouds.
In order to have accurate frequencies in the mm domain, we have performed a new set of measurements in our laboratory (in the $100-420 \mathrm{GHz}$ frequency range) for the $E$ and $Z$ isomers of $\mathrm{HNCHCN}$, which we present in this work, thus improving and enlarging the existing dataset of spectroscopic parameters.

Based on the spectroscopic results of this work, which allow us to provide accurate frequency predictions up to $700 \mathrm{GHz}$, we have carried out a search for HNCHCN emission toward a sample of 8 nearby (distances less than 250 pc) Sun-like-star forming regions in the earliest phases: from starless to more evolved Class 0 and I objects passing through Barnard 1, considered an hydrostatic core in a stage before the protostellar one (see Sect. 3). To this purpose, we used the ASAI unbiased highsensitivity spectral surveys at mm-wavelenghts. In summary, the main goal of the manuscript is twofold: (i) to improve the predictions for rotational transitions reaching $700 \mathrm{GHz}$ for both the $Z$ and $E$ isomers; and (ii) to search for HNCHCN (using the new frequencies) for the first time in a large sample of low-mass starforming regions.

\section{Experiment}

\subsection{Production of $C$ - cyanomethanimine}

C-cyanomethanimine is an unstable molecule that, in the present work, was produced by pyrolysis of dimethylcyanamide, $\left(\mathrm{CH}_{3}\right)_{2} \mathrm{NCN}$, as described by Takeo et al. (1986), by flowing the vapors of the precursor through a quartz tube heated by a $30 \mathrm{~cm}$ long tube furnace. The apparatus is the same used to produce other molecules of astrophysical interest, such as methanimine $\left(\mathrm{CH}_{2} \mathrm{NH}\right.$; Dore et al. 2012) and ketenimine $\left(\mathrm{CH}_{2} \mathrm{CNH}\right.$; Degli Esposti et al. 2014). The quartz reactor was connected to the usual gas inlet of the free-space absorption cell of the spectrometer and the pyrolysis products were pumped out continuously, but slowly, in order to provide their continuous flow inside the $3.25 \mathrm{~m}$ long, $10 \mathrm{~cm}$ diameter, glass cell.

For each isomer, the best working conditions were obtained by monitoring the absorption signal of a previously reported transition below $100 \mathrm{GHz}$ (from Takano et al. 1990). Slightly different optimal conditions were employed for two isomers: highest yields of $Z$-C-cyanomethanimine were obtained by setting the furnace temperature to $1100{ }^{\circ} \mathrm{C}$ and by flowing the precursor at a pressure of 60 mTorr through the quartz reactor, which corresponds to a pressure of 10 mTorr in the absorption cell. On the other hand, E-C-cyanomethanimine was found to have a higher production rate by using a lower pressure (20 mTorr in the quartz reactor) and a higher pyrolysis temperature $\left(1160^{\circ} \mathrm{C}\right)$.

\subsection{Millimeter/submillimeter-wave spectrometer}

The rotational spectra were recorded in the $100-117 \mathrm{GHz}$ and $240-419 \mathrm{GHz}$ frequency regions by means of a millimeter/submillimeter-wave frequency modulated spectrometer (Degli Esposti et al. 2017). Radiations sources are either a series of Gunn diodes covering the $75-134 \mathrm{GHz}$ range or passive multipliers driven by the Gunn diodes which extend the covered range from $225 \mathrm{GHz}$ to $1.2 \mathrm{THz}$. The output frequency is stabilized by a phase-lock loop (PLL) system referred to a signal of $75 \mathrm{MHz}$ and frequency modulated at $6 \mathrm{kHz}$. Phase sensitive detection at twice the modulation frequency is employed, so that the second derivative of the actual absorption profile is recorded. A Schottky barrier Millitech detector was used for recording 


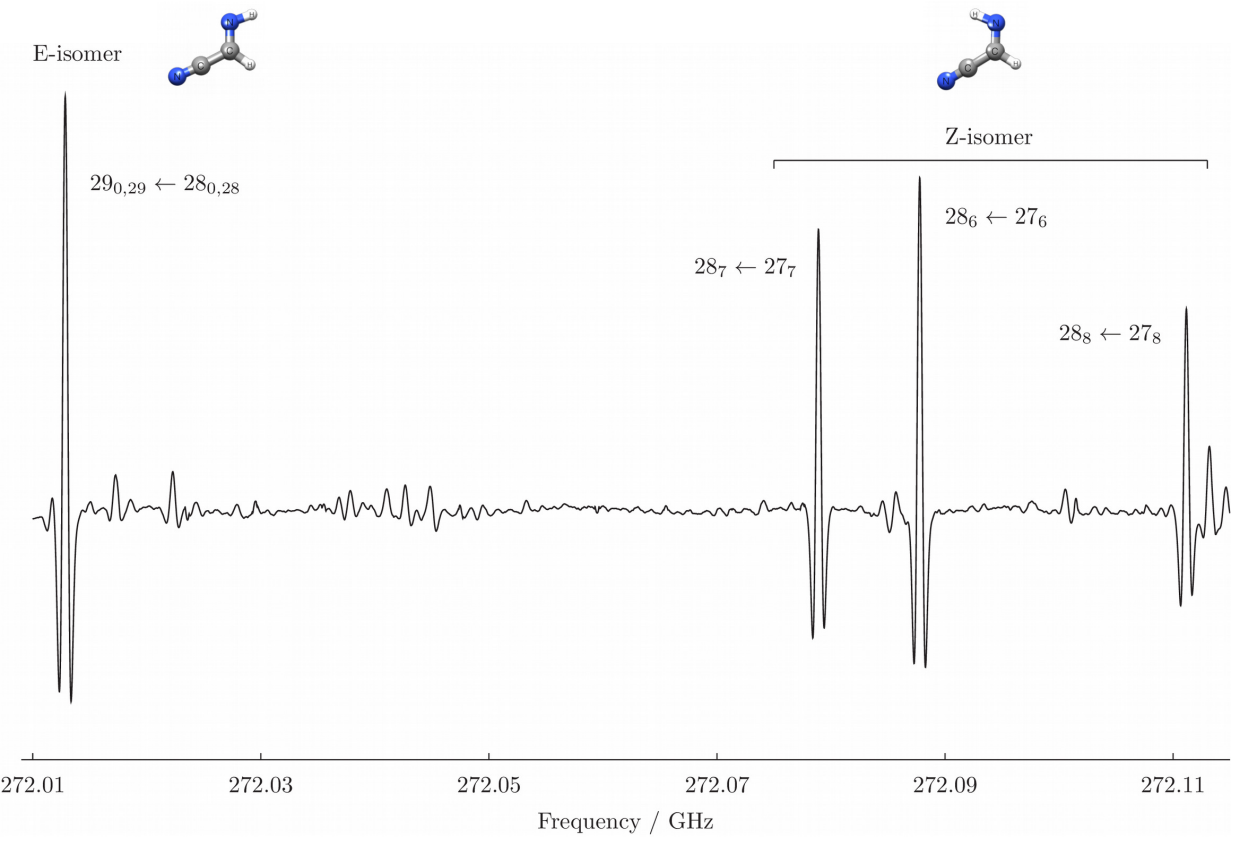

Fig. 1. Portion of the millimeter-wave spectrum, recorded in the best condition for the production of $E$ - cyanomethanimine. $a$-type $R$ branch transitions are visible for both isomers. In case of unresolved asymmetry doublets, only the $K_{a}$ subscript is reported. below $117 \mathrm{GHz}$, while VDI detectors were employed in the 240$419 \mathrm{GHz}$ region.

According to the experimental conditions, frequency range, and signal-to-noise ratio $(\mathrm{S} / \mathrm{N})$, the estimated uncertainties for our measurements range from 20 to $60 \mathrm{kHz}$. Figure 1 shows a small portion of spectra at $272 \mathrm{GHz}$. As seen, both isomers are present in the experimental mixture, with an abundance that ensures a very good $\mathrm{S} / \mathrm{N}$ of the spectrum. Despite the fact that the recording was carried out under the best conditions for producing the $E$ isomer and the lower dipole moment of $\mathrm{Z}-\mathrm{C}$ cyanomethanimine, the transitions appear to have similar intensity, indicating that the $Z$ isomer is produced in higher yield.

\section{The astronomical sample}

The data presented here are part of the Large Programme ASAI, which has collected unbiased spectral surveys using the Pico Veleta (Spain) 30-m single-dish. Observations and data reduction are reported in details in Lefloch et al. (in prep.). In summary, the observations were carried out in Wobbler Switching Mode, during several runs between 2012 and 2014 using the EMIR receivers at $3 \mathrm{~mm}(72-116 \mathrm{GHz}), 2 \mathrm{~mm}(126-173 \mathrm{GHz})$, and $1.3 \mathrm{~mm}(200-276 \mathrm{GHz})$. In the present context we searched for C-cyanomethanimine in different objects sampling different stages of the formation process leading to a Sun-like star, namely from starless cores to Class 0 and I objects, and in addition to jetdriven shocks regions. Below, a short description of the targets:

- L1544 is one of the best studied starless core located in Taurus at a distance of $140 \mathrm{pc}$ (see Caselli et al. 1999, 2002, and references within). The core is characterized by high molecular depletion given its high density $\left(\geq 2 \times 10^{6} \mathrm{~cm}^{-3}\right)$ and low temperature (down to $7 \mathrm{~K}$ ). Recently, emission due to the so-defined interstellar complex organic molecules (iCOMs) (e.g., $\mathrm{CH}_{3} \mathrm{CHO}, \mathrm{CH}_{3} \mathrm{OCHO}, \mathrm{CH}_{3} \mathrm{OCH}_{3}$ ) have been detected, plausibly coming from the external portion of the clouds due to reactive desorption and/or cosmic-rays irradiation (e.g., Vastel et al. 2014; Jiménez-Serra et al. 2016; Vasyunin et al. 2017, and references within).

- Barnard Blb is an active star-forming site (e.g., Bachiller \& Cernicharo 1986) located in the Barnard dense core, in Perseus $(d=235 \mathrm{pc})$. B 1b is associated with two companions, B1bN and B1bS: their spectral energy distribution (Pezzuto et al. 2012) and their association with compact and slow outflows (Gerin et al. 2015) make them among the best candidates for the first hydrostatic stage. In other words, Barnard B1b could be placed in an intermediate stage between starless cores and Class $0\left(\geq 10^{4} \mathrm{yr}\right)$ protostars. The observed position is associated with a rich molecular spectra containing for example, $\mathrm{CH}_{3} \mathrm{CHO}, \mathrm{CH}_{3} \mathrm{OCHO}$, and $\mathrm{CH}_{3} \mathrm{O}$ lines (e.g., Cernicharo et al. 2012; Daniel et al. 2013).

- IRAS4A is a binary Class 0 system in the Perseus NGC 1333 region, well identified using interferometry (e.g., Looney et al. 2000; Santangelo et al. 2015; Tobin et al. 2016, and references therein). The two objects, called A1 and A2, are separated by $1.8^{\prime \prime}$ (420 AU) and are associated with different properties: (i) IRAS4-A1 has an internal luminosity of $\sim 3 L_{\odot}$ (De Simone et al. 2017) and is more than three times brighter in the mm-flux than its companion; (ii) only IRAS4-A2 is associated with a emission due to iCOMs (e.g., $\mathrm{HCOCH}_{2} \mathrm{OH}$ among others; see Taquet et al. 2015; Coutens et al. 2015; De Simone et al. 2017). Both sources drive jets, with A1 definitely being the faster and younger of the two (Santangelo et al. 2015). The intrinsic different properties of jets and driving sources in NGC 1333-IRAS4A indicates different evolutionary stages, with $\mathrm{A} 2$ being evolved enough to develop a hot-corino region.

- L1157-mm is a Class 0 source with a bolometric luminosity of $\sim 3 L_{\odot}$ driving a precessing jet (Gueth et al. 1996, 1998; Podio et al. 2016), which, in turn, has created the prototype of the so-called chemically rich outflows (e.g., Bachiller et al. 2001). The protostar lies in a relatively isolated cloud in Cepheus, at a distance of $250 \mathrm{pc}$ (Looney et al. 2000), and is associated with an elongated molecular envelope possibly associated with a still not detected accretion disk (e.g., Gueth et al. 2003; Chiang 2012; Tobin et al. 2013a,b, and references therein).

- L1157-B1 is the brightest shocked region associated with the jet/outflow driven by the L1157-mm protostar. The jet has excavated two main cavities, with apices called B1 and B2 (Gueth et al. 1996, 1998). In particular, B1 has 
a kinematical age $\simeq 1100 \mathrm{yr}$ (Podio et al. 2016) and consists of a series of shocks caused by different episodes of ejection impacting against the cavity wall. L1157-B1 has been the target of several studies using single-dish and interferometric arrays revealing rich and intense molecular spectra (e.g., Tafalla \& Bachiller 1995; Bachiller et al. 2001; Codella et al. 2010; Lefloch et al. 2010; Nisini et al. 2010). Interestingly, high-angular resolution images revealed a chemical differentation indicating an active grain-surface chemistry at work (e.g., Codella et al. 2009; Benedettini et al. 2007, 2012, 2013; Busquet et al. 2014). Several iCOMs have been revealed, from the first detections reported by Arce et al. (2008) to the Lefloch et al. (2017) extensive survey, passing through the first detection of formamide in a shock (Mendoza et al. 2014).

- L1448 R2 is a shocked region located in the southern molecular outflow driven by a Class 0 protostar with a luminosity of about $7 L_{\odot}$ (e.g., Bachiller et al. 1990; De Simone et al. 2017). The region is located in Perseus, at the center of the L1448 complex (see Looney et al. 2000; Tobin et al. 2016, and references therein) at a distance of $232 \mathrm{pc}$ (Hirota et al. 2011). L1448 R2 has been studied in detail down to high-spatial resolutions revealing high-velocity bullets, high-excitation conditions, and a consequently enriched chemistry (e.g., $\mathrm{SiO}, \mathrm{H}_{2} \mathrm{O}, \mathrm{NH}_{3}$, high- $J$ CO lines: Dutrey et al. 1997; Nisini et al. 2010, 2013; Santangelo et al. 2012; Gómez-Ruiz et al. 2016). To our knowledge, no evidence of emission due to iCOMs has been found so far.

- $L 1527$ is a Class 0 source located in Taurus $(d=140 \mathrm{pc})$ with a bolometric luminosity of approximately $3 L_{\odot}$ (Tobin et al. 2013b, and references therein). The source is considered to be the prototypical warm carbon chain chemistry (WCCC) source, and is associated with an almost edge-on envelope/disk system (see e.g., Sakai et al. 2010, 2014b,a; Oya et al. 2015, and references therein). Recent ALMA images revealed the rich chemistry $\left(\mathrm{SO}, \mathrm{CH}_{3} \mathrm{OH}\right)$ activated by the slow shocks occurring at the centrifugal barrier of the infalling and rotating envelope (Oya et al. 2015; Sakai et al. 2017).

- SVS13-A is part of the system SVS13, located in the NGC 1333 cloud in Perseus at 235 pc from the Sun. In the $\mathrm{mm}$-spectral range the region is dominated by two protostars identified by interferometric observations (Bachiller et al. 1998; Looney et al. 2000; Chen et al. 2009; Tobin et al. 2016), called A and B, at 15" from each other. The internal luminosity of SVS13-A has been estimated to be around $25 L_{\odot}$ (De Simone et al. 2017). SVS13-A is associated with: (i) an extended ( $>0.07 \mathrm{pc}$ ) outflow; (ii) the HH711 chain (Lefloch et al. 1998, and references therein); and (iii) a low $L_{\text {submm }} / L_{\text {bol }}$ ratio $(\sim 0.8 \%)$. As a consequence, although SVS13-A is still deeply embedded in a largescale envelope ( $6000 \mathrm{AU}$; Lefloch et al. 1998), the protostar is considered a Class I ( $\left.\geq 10^{5} \mathrm{yr}\right)$ source (e.g., Chen et al. 2009, and references therein). Recently, the occurrence of a hot-corino around SVS13-A has been revealed through $\mathrm{HDO}$ and $\mathrm{HCOCH}_{2} \mathrm{OH}$ measurements (Codella et al. 2016; De Simone et al. 2017).

\section{Results}

\subsection{Spectroscopic parameters}

A total number of 286 and 311 new line frequencies were measured in the $100-419 \mathrm{GHz}$ range for the $E$ and $Z$ isomers, respectively. Our measurements involved rotational energy levels in the 10 to $46 J$ interval and ranging in $K_{a}$ from 0 to 15 . Small splittings due to the ${ }^{14} \mathrm{~N}$-nuclear spin of the two nitrogen atoms were observed for a few $a$ - and $b$-type $R$ branch transitions. The transition frequencies obtained in the present study together with those reported by Takeo et al. (1986) in the 37$50 \mathrm{GHz}$ range, by Takano et al. (1990) in the 23-100 GHz range, and by Zaleski et al. (2013) in the $9-48 \mathrm{GHz}$ range were analyzed using Pickett's SPFIT program (Pickett 1991), adopting Watson's S-reduced Hamiltonian in its $I^{r}$ representation (Watson 1977). Each transition frequency was weighted proportionally to the inverse square of its experimental uncertainty. The hyperfine structure exhibited by many lines of both isomers was accounted for using the following angular momenta coupling scheme between the ${ }^{14} \mathrm{~N}$-nuclear spins $\hat{I}_{1}$ (NH group) and $\hat{I}_{2}$ (CN group) and the rotational angular momentum $\hat{J}: \hat{I}=\hat{I}_{1}+\hat{I}_{2}, \hat{F}=\hat{J}+\hat{I}$. In this way, a total of 384 distinct transition frequencies were analyzed for each of the two isomers. These global fits led to the determination of 17 independent spectroscopic parameters with a root mean square (RMS) error of 36 and $38 \mathrm{kHz}$ and a dimensionless standard deviation of 0.81 and 0.95 for $Z$ - and $E$ C-cyanomethanimine, respectively. The results of the fits, compared to those by Zaleski et al. (2013), are collected in Table 1, while the list of frequencies is available in the supplementary material, which also contains the set of spectroscopic constants obtained in the SPFIT format (in order to facilitate their inclusion in spectroscopic databases). We note that, in addition to the improvement of the already known spectroscopic parameters, new constants have been determined. In particular, the quartic centrifugal distortion constant $D_{K}$ and four sextic centrifugaldistortion constants were obtained with good accuracy for both isomers. The ${ }^{14} \mathrm{~N}$ spin-rotation coupling constant $C_{a a}(\mathrm{NH})$ was also fitted. Overall, the accuracy of all spectroscopic constants previously reported has been improved by 1 to 3 order of magnitude. For both isomers, there is a noticeable difference in the newly determined $A$ rotational constant with respect to those of Zaleski et al. (2013), with differences of $\sim 5 \mathrm{MHz}$ for $E$-Ccyanomethanimine and $\sim 20 \mathrm{MHz}$ for the $Z$ isomer, the respective discrepancies being about 200 and 4 times the statistical errors given in Zaleski et al. (2013). These large changes are due to the fact that $b$-type transitions have been measured for the first time for Z-C-cyanomethanimine, while $b$-type transitions involving rotational levels with $K_{a}>1$ have been included in the fit for the $E$ isomer. Our global fits allowed us to improve the rest frequency determinations in terms of accuracy as well as to extend their availability up to $700 \mathrm{GHz}$ with uncertainty smaller than $200 \mathrm{kHz}$. By means of a selection of observed transition frequencies together with the corresponding residuals from the fits, Table 2 provides the reader with an example of the quality of the measurements and analysis for both isomeric species.

Figures 2 and 3 provide an overview of the rotational spectra in the $0-1000 \mathrm{GHz}$ frequency range for the $E$ and $Z$ isomers, respectively, at four different temperatures: $T=10 \mathrm{~K}$ (upper row), $T=30 \mathrm{~K}$ (second row), $T=100 \mathrm{~K}$ (third row), and $T=300 \mathrm{~K}$ (lower row). For both isomers, it is observed that by increasing the temperature the maximum of intensity moves to higher frequencies. The overall spectra also reflect the dipole moment components of the two species. For E-C-cyanomethanimine, both $a$ - and $b$-type spectra are intense (see Fig. 2), as expected by the large dipole moment components. However, the $b$-type is the most intense at all temperatures considered, even if at $T=300 \mathrm{~K}$ the $a$-type transitions become nearly as intense as the $b$-type ones (see Fig. 2, lower row). For the $Z$ isomer, the $a$-type spectrum is the more intense independently of the temperature because of the 
M. Melosso et al.: C-cyanomethanimine: laboratory and astronomical measurements

Table 1. Spectroscopic parameters determined for the $\mathrm{Z}$ and $\mathrm{E}$ isomers of $\mathrm{C}$-cyanomethanimine.

\begin{tabular}{|c|c|c|c|c|c|}
\hline \multirow[b]{2}{*}{ Parameters } & & \multicolumn{2}{|c|}{ E-C-cyanomethanimine } & \multicolumn{2}{|c|}{ Z-C-cyanomethanimine } \\
\hline & & Present work & Previous results $^{a}$ & Present work & Previous results ${ }^{a}$ \\
\hline$A$ & & $62700.392(22)^{b}$ & $62695.094(24)$ & $54193.405(32)$ & $54173.1(50)$ \\
\hline$B$ & & $4972.04534(22)$ & $4972.04643(81)$ & $5073.86584(15)$ & $5073.86506(86)$ \\
\hline$C$ & & $4600.29561(23)$ & $4600.29460(89)$ & $4632.38905(14)$ & $4632.39090(74)$ \\
\hline$D_{J}$ & $\times 10^{3}$ & $1.881477(94)$ & $1.8704(55)$ & $2.425671(87)$ & $2.4737(74)$ \\
\hline$D_{J K}$ & & $-0.1054288(30)$ & $-0.10455(17)$ & $-0.1032838(27)$ & $-0.10331(21)$ \\
\hline$D_{K}$ & & $5.1408(52)$ & & $3.5488(59)$ & \\
\hline$d_{1}$ & $\times 10^{3}$ & $-0.33939(13)$ & $-0.3272(57)$ & $-0.48265(10)$ & $-0.4961(82)$ \\
\hline$d_{2}$ & $\times 10^{3}$ & $-0.020511(20)$ & $0.0221(71)$ & $-0.031338(21)$ & $-0.0321(55)$ \\
\hline$H_{J}$ & $\times 10^{9}$ & $4.661(33)$ & & $6.998(35)$ & \\
\hline$H_{J K}$ & $\times 10^{6}$ & $-0.42172(97)$ & & $-0.40281(93)$ & \\
\hline$H_{K J}$ & $\times 10^{6}$ & $-7.303(11)$ & & $-8.986(15)$ & \\
\hline$H_{K}$ & $\times 10^{3}$ & $0.906723^{c}$ & & $0.599872^{c}$ & \\
\hline$h_{1}$ & $\times 10^{9}$ & $1.823(45)$ & & $2.555(38)$ & \\
\hline$h_{2}$ & $\times 10^{9}$ & $0.1571^{c}$ & & $0.2675^{c}$ & \\
\hline$h_{3}$ & $\times 10^{9}$ & $0.0450^{c}$ & & $0.0738^{c}$ & \\
\hline$\chi_{a a}(C N)$ & & $-4.1315(20)$ & $-4.1280(67)$ & $-4.0102(51)$ & $-4.012(21)$ \\
\hline$\chi_{b b}(C N)$ & & $-0.2006(31)$ & $-0.1972(57)$ & $-0.20845(39)$ & $-0.2146(86)$ \\
\hline$\chi_{a a}(N H)$ & & $0.7447(30)$ & $0.753(11)$ & $-4.2721(49)$ & $-4.269(21)$ \\
\hline$\chi_{b b}(N H)$ & & $-2.0661(51)$ & $-2.0642(89)$ & $-0.81008(50)$ & $-0.8201(82)$ \\
\hline$C_{a a}(N H)$ & & $0.0173(19)$ & & $0.0064(22)$ & \\
\hline \# lines & & 384 & & 384 & \\
\hline RMS error & & 0.036 & & 0.038 & \\
\hline$\sigma$ & & 0.81 & & 0.95 & \\
\hline
\end{tabular}

Notes. Units are in MHz, except the dimensionless standard deviation $\sigma .{ }^{(a)}$ Zaleski et al. (2013). ${ }^{(b)}$ Values in parenthesis denote one standard deviation and apply to the last digits of the constants. ${ }^{(c)}$ Fixed at the computed value (Puzzarini 2015).

Table 2. Selection of observed transition frequencies together with the corresponding residuals from the fits for the $Z$ and $E$ isomers of Ccyanomethanimine.

\begin{tabular}{|c|c|c|c|c|c|c|c|c|c|}
\hline Isomer & $\begin{array}{l}J^{\prime} \\
\mathrm{Upp}\end{array}$ & $\begin{array}{l}K_{a}^{\prime} \\
\text { r st }\end{array}$ & $e_{c}^{K_{c}^{\prime}}$ & $\begin{array}{c}J \\
\text { Low }\end{array}$ & $\begin{array}{l}K_{a} \\
\text { er st }\end{array}$ & $K_{c}$ & $\begin{array}{c}\text { Obs. frequency } \\
(\mathrm{MHz})\end{array}$ & $\begin{array}{c}\text { Uncertainty } \\
(\mathrm{MHz})\end{array}$ & $\begin{array}{l}\text { Obs.-Calc. } \\
\text { (MHz) }\end{array}$ \\
\hline$(Z)$ & 30 & 3 & 28 & 29 & 3 & 27 & 291623.715 & 0.030 & 0.008 \\
\hline$(Z)$ & 30 & 5 & 26 & 29 & 5 & 25 & 291640.038 & 0.030 & -0.018 \\
\hline$(Z)$ & 30 & 5 & 25 & 29 & 5 & 24 & 291646.827 & 0.030 & -0.007 \\
\hline$(Z)$ & 30 & 4 & 27 & 29 & 4 & 26 & 291842.010 & 0.030 & -0.012 \\
\hline$(Z)$ & 30 & 4 & 26 & 29 & 4 & 25 & 292014.364 & 0.030 & -0.051 \\
\hline$(E)$ & 42 & 2 & 41 & 42 & 1 & 42 & 345022.426 & 0.030 & -0.006 \\
\hline$(E)$ & 36 & 5 & 31 & 35 & 5 & 30 & 345027.550 & 0.040 & 0.008 \\
\hline$(E)$ & 36 & 9 & 27 & 35 & 9 & 26 & 345039.468 & 0.040 & $0.000^{(a)}$ \\
\hline$(E)$ & 36 & 9 & 28 & 35 & 9 & 27 & 345039.468 & 0.040 & $0.000^{(a)}$ \\
\hline$(E)$ & 37 & 0 & 37 & 36 & 0 & 36 & 345190.487 & 0.040 & 0.006 \\
\hline
\end{tabular}

Notes. ${ }^{(a)}$ Blended transitions: relative weight 0.5 .

small magnitude of $\mu_{b}$ (see Fig. 3). Despite the fact that the $b$ type spectrum is particularly weak, in this work, for the first time, $b$-type transitions have been measured and the observed intensities tend to confirm qualitatively a value of $0.4 \mathrm{D}$ for $\mu_{b}$. Overall, the rotational spectrum of $Z$-C-cyanomethanimine is weaker than that of the $E$ isomer by about one order of magnitude. An example is shown in Fig. 4, which allows us to point out the good $\mathrm{S} / \mathrm{N}$ of our measurements.

\subsection{Astronomical observations: abundance upper limits}

To obtain the most constraining information on the presence of $\mathrm{C}$-cyanomethanimine in the selected astronomical targets, we used the frequencies (falling in the ASAI spectral ranges) of the $E$ isomer, whose transitions are always brighter than those of the $Z$ one. Based on the predictions showed of Fig. 2, and conservatively homogenising the search for $\mathrm{HNCHCN}$ in the present sample, we selected the brightest lines assuming a temperature (and the corresponding partition function) of $10 \mathrm{~K}$ for the starless core L1544 and for Barnard 1, while we used a representative temperature of $100 \mathrm{~K}$ for the regions around protostars and outflow shocks.

Figure 2 clearly shows that the most intense lines at $10 \mathrm{~K}$ fall in the $3 \mathrm{~mm}$ spectral window (where the Half Power Beam Width, HPBW, is $\sim 26^{\prime \prime}$ ), while at $100 \mathrm{~K}$ they lie at $1.3 \mathrm{~mm}$ $\left(H P B W \sim 12^{\prime \prime}\right)$. As an example, the $9_{0,9}-8_{0,8}$ transition at 

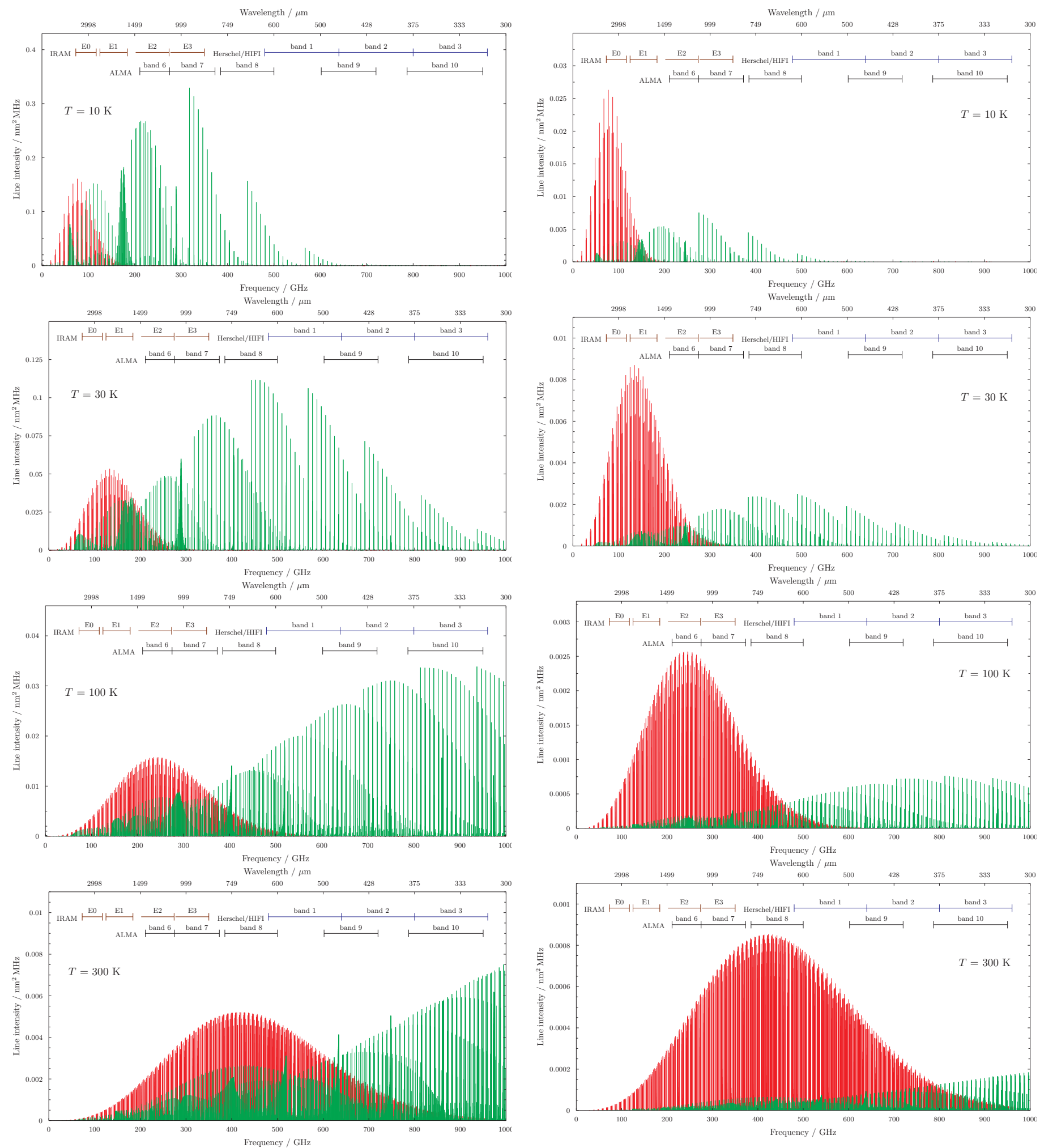

Fig. 2. Spectral predictions in the $0-1000 \mathrm{GHz}$ frequency range for the $E$-C-cyanomethanimine at four different temperatures: $T=10 \mathrm{~K}$ (upper row), $T=30 \mathrm{~K}$ (second row), $T=100 \mathrm{~K}$ (third row), and $T=300 \mathrm{~K}$ (lower row). $a$-type transitions are depicted in red, $b$-type transitions in green.

$85931.777 \mathrm{MHz}$ with $E_{\mathrm{u}}=115 \mathrm{~K}$ and $S \mu^{2}=233 \mathrm{D}^{2}$ is expected to be one of the brightest lines at $10 \mathrm{~K}$. On the other hand, a good candidate at $100 \mathrm{~K}$ is the $22_{0,22}-21_{0,21}$ transition at $207705.762 \mathrm{MHz}\left(E_{\mathrm{u}}=21 \mathrm{~K}, S \mu^{2}=190 \mathrm{D}^{2}\right)$. Unfortunately,

Fig. 3. Spectral predictions in the $0-1000 \mathrm{GHz}$ frequency range for the $Z$-C-cyanomethanimine at four different temperatures: $T=10 \mathrm{~K}$ (upper row), $T=30 \mathrm{~K}$ (second row), $T=100 \mathrm{~K}$ (third row), and $T=300 \mathrm{~K}$ (lower row). $a$-type transitions are depicted in red, $b$-type transitions in green.

no lines due to C-cyanomethanimine were detected. However, thanks to the high-sensitivity of the ASAI dataset, we derived constraining (see below) upper limits on the HNCHCN column density in low-mass star-forming regions. To obtain the best 
sensitivity, we smoothed the spectral resolution of the L1544 spectra to $0.5 \mathrm{~km} \mathrm{~s}^{-1}$, given the expected linewidth of iCOMs lines is between $0.3 \mathrm{~km} \mathrm{~s}^{-1}$ and $0.8 \mathrm{~km} \mathrm{~s}^{-1}$ (Vastel et al. 2014). On the other hand, we smoothed the spectra of Barnard 1, protostars and shocks to $2 \mathrm{~km} \mathrm{~s}^{-1}$ because we expect a linewidth of at least $4 \mathrm{~km} \mathrm{~s}^{-1}$ (e.g., De Simone et al. 2017; Lefloch et al. 2017; Cernicharo et al. 2012). The $1 \sigma$ level of the integrated area (in main-brightness temperature scale, $T_{\mathrm{MB}}$ ) of the lines are: $\sim 10$ $20 \mathrm{mK} \mathrm{km} \mathrm{s}^{-1}$ for L1544 and Barnard1, and $\simeq 30-90 \mathrm{mK} \mathrm{km} \mathrm{s}^{-1}$ for the other targets. Using a $3 \sigma$ level criterium, we obtained the following beam averaged (12" for all the sources but L1544 and Barnard 1, averaged on 26" ) upper limits:

$$
\begin{aligned}
& -1 \times 10^{11} \mathrm{~cm}^{-2}(\text { L1544; 26"); } \\
& -2 \times 10^{11} \mathrm{~cm}^{-2}\left(\text { Barnard } 1 ; 26^{\prime \prime}\right) ; \\
& -1 \times 10^{12} \mathrm{~cm}^{-2}(\text { L1157-B1; 12" }) ; \\
& -2 \times 10^{12} \mathrm{~cm}^{-2}(\text { L1157-mm, L1448-R2; 12" }) ; \\
& -3 \times 10^{12} \mathrm{~cm}^{-2}\left(\text { IRAS4A, L1527, SVS13-A; } 12^{\prime \prime}\right) .
\end{aligned}
$$

An estimate of the upper limits for the cyanomethanimine abundance, $X_{\mathrm{HNCHCN}}$, can be obtained using the $\mathrm{H}_{2}$ column density: namely $\mathrm{N}\left(\mathrm{H}_{2}\right) \simeq 10^{23} \mathrm{~cm}^{-2}$ for the starless core L1544 (Crapsi et al. 2005), $\simeq 10^{21} \mathrm{~cm}^{-2}$ for the L1157-B1 shock (Lefloch et al. 2012), and around $10^{24} \mathrm{~cm}^{-2}$ for the hot-corinos associated with SVS13-A and IRAS4A (e.g., Looney et al. 2000; Maret et al. 2002; Codella et al. 2010). For a proper comparison, the beam averaged $N_{\mathrm{HNCHCN}}$ upper limits have to be first modified taking into account the expected emitting size, being consequently corrected for the corresponding beam dilution. For hot-corinos, we assumed a typical size of $1^{\prime \prime}$ and then the filling factor ${ }^{7} f f=7 \times 10^{-3}$; for the L1157-B1 shock, we used $9^{\prime \prime}$ and $f f=0.36$ (Lefloch et al. 2012). A filling factor $f f=1$ (i.e., no correction) has been assumed instead for L1154 given its extended structure (e.g., Vastel et al. 2014). Therefore, by assuming that $\mathrm{HNCHCN}$ and $\mathrm{H}_{2}$ are tracing the same material and comparing the corresponding column densities, we derived $X_{\mathrm{HNCHCN}} \leq 4 \times 10^{-10}$ for starless and hot-corinos, and $\leq 5 \times 10^{-9}$ for shocks.

\subsection{Discussion}

As stated in the Introduction, the unique detection of cyanomethanimine in the interstellar medium so far has been reported by Zaleski et al. (2013), who observed emission due to the $E$ isomer toward the $\mathrm{B} 2(\mathrm{~N})$ core of the Sagittarius complex. Sagittarius B2(N) can be considered one of the best places where to search for complex organic molecules (e.g., Belloche et al. 2009). The Sagittarius B2 region is one of the largest molecular clouds in the Galaxy associated with massive star-forming regions, and it is located at about $120 \mathrm{pc}$ from the Galactic Center. Zaleski et al. (2013) derived a $N_{\mathrm{HNCHCN}} \simeq 10^{13} \mathrm{~cm}^{-2}$ using lines observed at $1 \mathrm{~cm}$ using the Green Bank Telescope (GBT), but no abundance has been calculated. This is plausibly due to the uncertainty on the size of the emitting region, Sgr B2(N) being a source with a substantial structure on spatial scales smaller than the GBT beam (from $\simeq 20^{\prime \prime}$ to $80^{\prime \prime}$, depending on the frequency, i.e. $\simeq 1-3 \mathrm{pc}$ given the $\mathrm{Sgr} \mathrm{B} 2(\mathrm{~N})$ distance). As a consequence, it is not clear which is the $\mathrm{H}_{2}$ column density of the observed features: if compact, $N_{\mathrm{H}_{2}}$ is surely larger than $10^{24} \mathrm{~cm}^{-2}$, which in turn means $X_{\mathrm{HNCHCN}} \leq 10^{-11}$. However, we cannot exclude that the HNCHCN lines in Sgr B2(N) arise from the external layers of the cloud where $N_{\mathrm{H}_{2}}$ could be lower.

$f f=\theta_{\mathrm{s}}^{2} /\left(\theta_{\mathrm{s}}^{2}+\theta_{\mathrm{b}}^{2}\right)$, where $\theta_{\mathrm{s}}$ and $\theta_{\mathrm{b}}$ are the source and the beam sizes, respectively.
The advantages of the upper limits on $X_{\mathrm{HNCHCN}}$ presented here are the following: (i) they refer to regions associated with Sun-like progenitors which are expected to be associated with protoplanetary regions; and (ii) previous observations of the astronomical sample make one confident to give a reasonable assumption on the cyanomethanimine emitting region.

Interestingly, the present upper limits on $X_{\mathrm{HNCHCN}}$ can be compared with the abundance of another N-bearing iCOMs, such as formamide $\left(\mathrm{NH}_{2} \mathrm{CHO}\right)$, measured by Mendoza et al. (2014) and López-Sepulcre et al. (2015) toward shocks $\left(X_{\mathrm{NH}_{2} \mathrm{CHO}}=5 \times 10^{-9}\right)$ and hot-corinos $\left(3 \times 10^{-11}\right.$ and 2$5 \times 10^{-10}$ for SVS13-A and IRAS4A, respectively). As a consequence, we have $R=X_{\mathrm{HNCHCN}} / X_{\mathrm{NH}_{2} \mathrm{CHO}} \leq 10$ (SVS13A), while for L1157-B1 and IRAS4A we have one order of magnitude less: $R \leq 1$. Although these measurements do not severely constrain the ratio between cyanomethanimine and formamide around Sun-like-star forming regions, they are in agreement with what we can derive from the column densities reported toward the massive star-forming region Sgr B2(N) by Zaleski et al. (2013) and Halfen et al. (2011). With the same caveats reported above on the use of large beams around $\mathrm{Sgr}$ $\mathrm{B} 2(\mathrm{~N}): N_{\mathrm{HNCHCN}}=2 \times 10^{13} \mathrm{~cm}^{-2}, N_{\mathrm{NH}_{2} \mathrm{CHO}}=4 \times 10^{14} \mathrm{~cm}^{-2}$, and thus $R \sim 0.1$.

\section{Conclusions}

In the present paper, the investigation of the rotational spectrum of C-cyanomethanimine has been extended to the millimeter/submillimeter-wave frequency region, thus considering the 100-419 GHz range. New measurements, which also include the first recording of $b$-type transitions for the $Z$ isomer, have allowed us to improve and enlarge the sets of spectroscopic parameters. Overall, the present work is able to provide accurate predictions for rotational transitions up to $700 \mathrm{GHz}$ for both $Z$ - and E-C-cyanomethanimine. This is an important prebiotic species, whose $E$ form has already been detected in $\operatorname{SgB} 2(\mathrm{~N})$ at frequencies below $48 \mathrm{GHz}$. The extension to higher frequencies provided by this work opens up the opportunity of also detecting the $Z$ isomer, which has an $a$-type rotational spectrum weaker than that of $E$-C-cyanomethanimine by a factor of six, thus being too weak below $50 \mathrm{GHz}$ to be observed.

Using the frequencies here derived, we performed a search for C-cyanomethanimine emission toward nearby Sun-like-star forming regions using the ASAI IRAM 30-m dataset. We investigated the earliest stages of the star forming process, from starless regions to the more evolved hot-corinos associated with both Class 0 and Class I objects. We sampled spatial scales between 1600 and $2800 \mathrm{AU}$, depending on the targets, obtaining the following upper limits on the C-cyanomethanimine column density: $\sim 10^{11} \mathrm{~cm}^{-2}$ for the starless core L1544, and fews $10^{12} \mathrm{~cm}^{-2}$ for the hydrostatic core Barnard 1, hot-cores, and shocked regions. Using previous knowledge on the $\mathrm{H}_{2}$ column densities of the observed regions, we derived $\mathrm{C}$-cyanomethanimine abundances less than a few $10^{-10}$ and $10^{-9}$ for starless and hotcorinos, and shocks, respectively. Finally, the present constraints on the C-cyanomethanimine abundance could be used as instructive limits on the abundances in the gas-phase of prebiotic N-bearing molecules more complex than HNCHCN, such as glycine.

Acknowledgements. This work has been supported in Bologna by MIUR "PRIN 2015" funds (project "STARS in the CAOS (Simulation Tools for Astrochemical Reactivity and Spectroscopy in the Cyberinfrastructure for Astrochemical Organic Species)" - Grant Number 2015F59J3R) and by the University of 


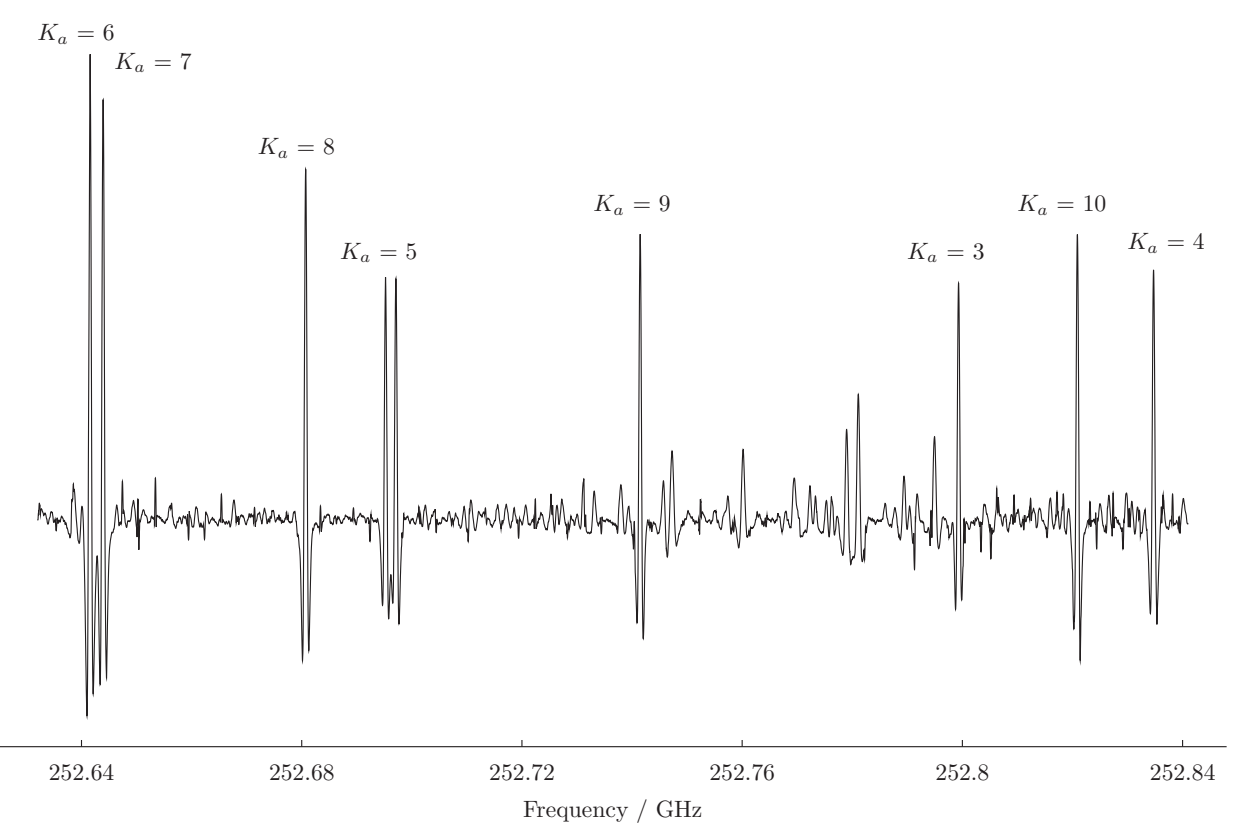

Fig. 4. Portion of the $J=26 \leftarrow 25 a$-type band of $Z$-cyanomethanimine. Both components of the asymmetry doublets are visible for the $K_{a}=5$ transition. for $K_{a}=3$ and $K_{a}=4$ transitions, only the low-frequency component is visible, while the asymmetry doublet is not resolved for all the other transitions.
Bologna (RFO funds). This work has also been partially supported by the PRININAF 2016 "The Cradle of Life - GENESIS-SKA (General Conditions in Early Planetary Systems for the rise of life with SKA)". The support of the COST CMTS-Actions CM1405 (MOLIM: MOLecules In Motion) and CM1401 (Our Astro-Chemical History) is acknowledged. C.C. acknowledges the funding from the European Research Council (ERC), project DOC (contract 741002). J.C. thanks ERC for funding under ERC-2013-SyG, G.A. 610256 NANOCOSMOS.

\section{References}

Arce, H. G., Santiago-García, J., Jørgensen, J. K., Tafalla, M., \& Bachiller, R. 2008, ApJ, 681, L21

Bachiller, R., \& Cernicharo, J. 1986, A\&A, 166, 283

Bachiller, R., Guilloteau, S., Gueth, F., et al. 1998, A\&A, 339, L49

Bachiller, R., Martin-Pintado, J., Tafalla, M., Cernicharo, J., \& Lazareff, B. 1990, A\&A, 231, 174

Bachiller, R., Gutiérrez, M. P., Kumar, M., \& Tafalla, M. 2001, A\&A, 372, 899 Balucani, N. 2009, Int. J. Mol. Sci., 10, 2304

Belloche, A., Garrod, R. T., Müller, H. S. P., et al. 2009, A\&A, 499, 215

Benedettini, M., Viti, S., Codella, C., et al. 2007, MNRAS, 381, 1127

Benedettini, M., Busquet, G., Lefloch, B., et al. 2012, A\&A, 539, L3

Benedettini, M., Viti, S., Codella, C., et al. 2013, MNRAS, 436, 179

Busquet, G., Lefloch, B., Benedettini, M., et al. 2014, A\&A, 561, A120

Caselli, P., Walmsley, C., Tafalla, M., Dore, L., \& Myers, P. 1999, ApJ, 523, L165

Caselli, P., Walmsley, C., Zucconi, A., et al. 2002, ApJ, 565, 344

Cernicharo, J., Marcelino, N., Roueff, E., et al. 2012, ApJ, 759, L43

Chen, X., Launhardt, R., \& Henning, T. 2009, ApJ, 691, 1729

Chiang, H. 2012, ApJ, 709, 470

Codella, C., Benedettini, M., Beltrán, M., et al. 2009, A\&A, 507, L25

Codella, C., Lefloch, B., Ceccarelli, C., et al. 2010, A\&A, 518, L112

Codella, C., Ceccarelli, C., Bianchi, E., et al. 2016, MNRAS, 462, L75

Coutens, A., Persson, M. V., Jørgensen, J. K., Wampfler, S. F., \& Lykke, J. M. 2015, A\&A, 576, A5

Crapsi, A., Caselli, P., Walmsley, C. M., et al. 2005, ApJ, 619, 379

Daniel, F., Gérin, M., Roueff, E., et al. 2013, A\&A, 560, A3

De Simone, M., Codella, C., Testi, L., et al. 2017, A\&A, 599, A121

Degli Esposti, C., Dore, L., \& Bizzocchi, L. 2014, A\&A, 565, A66

Degli Esposti, C., Dore, L., Melosso, M., et al. 2017, ApJS, 230, 7

Dore, L., Bizzocchi, L., \& Degli Esposti, C. 2012, A\&A, 544, A19

Dutrey, A., Guilloteau, S., \& Bachiller, R. 1997, A\&A, 325, 758

Evans, R. A., Lorencak, P., Ha, T. K., \& Wentrup, C. 1991, JACS, 113, 7261

Ferris, J. P., \& Hagan, W. J. 1984, Tetrahedron, 40, 1093
Gerin, M., Pety, J., Fuente, A., et al. 2015, A\&A, 577, L2

Gómez-Ruiz, A., Codella, C., Viti, S., et al. 2016, MNRAS, 462, 2203

Gueth, F., Guilloteau, S., \& Bachiller, R. 1996, A\&A, 307, 891

Gueth, F., Guilloteau, S., \& Bachiller, R. 1998, A\&A, 333, 287

Gueth, F., Bachiller, R., \& Tafalla, M. 2003, A\&A, 401, L5

Halfen, D. T., Ilyushin, V., \& Ziurys, L. M. 2011, ApJ, 743, 60

Hirota, T., Honma, M., Imai, H., et al. 2011, PASJ, 63, 1

Jiménez-Serra, I., Vasyunin, A. I., Caselli, P., et al. 2016, ApJ, 830, L6

Lefloch, B., Castets, A., Cernicharo, J., Langer, W., \& Zylka, R. 1998, A\&A, 334, 269

Lefloch, B., Cabrit, S., Codella, C., et al. 2010, A\&A, 518, L113

Lefloch, B., Cabrit, S., Busquet, G., et al. 2012, ApJ, 757, L25

Lefloch, B., Ceccarelli, C., Codella, C., et al. 2017, MNRAS, 469, L73

Looney, L. W., Mundy, L. G., \& Welch, W. 2000, ApJ, 529, 477

López-Sepulcre, A., Jaber, A. A., Mendoza, E., et al. 2015, MNRAS, 449, 2438

Maret, S., Ceccarelli, C., Caux, E., Tielens, A. G. G. M., \& Castets, A. 2002, A\&A, 395, 573

Mendoza, E., Lefloch, B., López-Sepulcre, A., et al. 2014, MNRAS, 445, 151

Müller, H. S. P., Schlöder, F., Stutzki, J., \& Winnewisser, G. 2005, J. Mol. Struct., 742,215

Nisini, B., Benedettini, M., Codella, C., et al. 2010, A\&A, 518, L120

Nisini, B., Santangelo, G., Antoniucci, S., et al. 2013, A\&A, 549, A16

Oya, Y., Sakai, N., Lefloch, B., et al. 2015, ApJ, 812, 59

Pezzuto, S., Elia, D., Schisano, E., et al. 2012, A\&A, 547, A54

Pickett, H. M. 1991, J. Mol. Spectr., 148, 371

Podio, L., Codella, C., Gueth, F., et al. 2016, A\&A, 593, L4

Puzzarini, C. 2015, J. Phys. Chem. A, 119, 11614

Sakai, N., Sakai, T., Hirota, T., \& Yamamoto, S. 2010, ApJ, 722, 1633

Sakai, N., Oya, Y., Sakai, T., et al. 2014a, ApJ, 791, L38

Sakai, N., Sakai, T., Hirota, T., et al. 2014b, Nature, 507, 78

Sakai, N., Oya, Y., Higuchi, A. E., et al. 2017, MNRAS, 467, L76

Santangelo, G., Nisini, B., Giannini, T., et al. 2012, A\&A, 538, A45

Santangelo, G., Codella, C., Cabrit, S., et al. 2015, A\&A, 584, A126

Tafalla, M., \& Bachiller, R. 1995, ApJ, 443, L37

Takano, S., Sugie, M., Sugawara, K.-i., et al. 1990, J. Mol. Spectr., 141, 13

Takeo, H., Sugie, M., Matsumura, C., Hamada, Y., \& Tsuboi, M. 1986, CPL, 123,229

Taquet, V., López-Sepulcre, A., Ceccarelli, C., et al. 2015, ApJ, 804, 81

Tobin, J. J., Bergin, E. A., Hartmann, L., et al. 2013a, ApJ, 765, 18

Tobin, J. J., Chandler, C. J., Wilner, D. J., et al. 2013b, ApJ, 779, 93

Tobin, J. J., Looney, L. W., Li, Z.-Y., et al. 2016, ApJ, 818, 73

Vastel, C., Ceccarelli, C., Lefloch, B., \& Bachiller, R. 2014, ApJ, 795, L2

Vasyunin, A., Caselli, P., Dulieu, F., \& Jiménez-Serra, I. 2017, ApJ, 842, 18

Watson, J. K. 1977, Vibrational spectra and structure, 6, 1

Zaleski, D. P., Seifert, N. A., Steber, A. L., et al. 2013, ApJ, 765, L10 